\section{CD147 est un récepteur essentiel à la colonisation vasculaire par le méningocoque}

Philippe C. Morand ${ }^{1-3}$, Nawal Maïssa ${ }^{1-3}$, Sandra C. Bernard ${ }^{1-4}$, Sandrine Bourdoulous ${ }^{1-3}$
${ }^{1}$ Inserm U1016, Institut Cochin, 22, rue Méchain, 75014 Paris, France ;

${ }^{2}$ CNRS, UMR8104, Paris, France ;

${ }^{3}$ université Paris Descartes, Sorbonne Paris Cité, Paris, France ;

${ }^{4}$ université Paris Diderot, Paris, France. sandrine.bourdoulous@inserm.fr
$>$ Les infections invasives à Neisseria meningitidis (méningocoque), responsables de septicémies et de méningites, sont une préoccupation de santé publique, à la fois dans les pays industrialisés et les pays émergents, en raison du taux de mortalité élevé, de la gravité des séquelles et du caractère parfois foudroyant de l'infection, même en cas de prise en charge médicale et d'antibiothérapie précoces [1, 2]. Alors que le méningocoque est présent de manière asymptomatique au niveau rhinopharyngé chez un grand nombre de sujets sains, la pathologie survient lorsque, dans des circonstances mal élucidées, la bactérie franchit la barrière oropharyngée et fait irruption dans la circulation sanguine pour s'y multiplier et disséminer dans différents organes (cerveau, peau, articulation, etc.) (Figure I). La colonisation de l'endothélium vasculaire par le méningocoque est l'étape préalable au franchissement des barrières endothéliales et à l'envahissement de l'espace périvasculaire dans les différents organes touchés [3, 4]. Cette interaction intime s'accompagne d'une coagulation intravasculaire et d'une altération des vaisseaux pouvant aller jusqu'à leur rupture dans les formes les plus graves (purpura fulminans).

\section{Les pili du méningocoque}

Chez les isolats pathogènes de méningocoques, généralement capsulés, les pili de type IV constituent le principal attribut bactérien permettant l'adhérence aux cellules endothéliales [3] (Figure IA). Ces pili sont des structures filamenteuses constituées d'une sous-unité majoritaire, la piline Pilk, et de plusieurs pilines « mineures» par leur abondance (PilV, PilX, ComP) mais associées à différentes fonctions spécifiques, respectivement adhérence aux cellules humaines, agrégation des pili et acquisition d'ADN exogène autologue (compétence) [5]. L'adhérence du méningocoque aux cellules endothéliales s'accompagne de l'activation de cascades de signalisation déclenchées par l'interaction des constituants Pilદ et PilV avec un récepteur cellulaire, le récepteur $\beta 2$-adrénergique $[6,7]$. Cependant, l'absence de ce récepteur cellulaire de signalisation n'empêche pas l'adhérence du méningocoque, ce qui suggérait l'existence d'un autre récepteur spécifiquement responsable de l'adhérence initiale du méningocoque. Notre équipe a récemment identifié CD147, également appelé Basigin ou extracellular matrix metalloproteinase inducer (EMPRINN), comme un récepteur indispensable à l'adhérence initiale du méningocoque aux cellules endothéliales périphériques et cérébrales [8] (Figure 2).

Le récepteur endothélial CD147 permet l'adhérence initiale du méningocoque aux cellules endothéliales

La découverte de CD147 comme candidat récepteur cellulaire pour l'adhérence initiale du méningocoque est issue d'une analyse transcriptionnelle différentielle sur 10478 gènes, réalisée à partir de cellules endothéliales humaines dédifférenciées rendues permissives à l'adhérence du méningocoque après traitement par un activateur transcriptionnel. Parmi 211 gènes dont l'expression était induite dans les cellules permissives, CD147, un membre de la superfamille des immunoglobulines (Ig) [9], est apparu comme un bon candidat récepteur pour l'adhérence du méningocoque aux cellules endothéliales car il réunissait plusieurs conditions attendues pour cette fonction : (1) CD147 est fortement exprimé sur les cellules endothéliales des microcapillaires cérébraux auxquels le méningocoque adhère préférentiellement; (2) CD147 s'accumule dès les premières minutes aux sites d'infection du méningocoque sur la membrane cellulaire; et (3) son recrutement est indépendant de l'expression du récepteur $\beta 2$-adrénergique, suggérant qu'il intervient en amont de l'activation de voies de signalisation.

Plusieurs approches complémentaires in vitro ont permis de confirmer le rôle du CD147 dans l'adhérence du méningocoque aux cellules endothéliales humaines. La déplétion en CD147, la présence de formes solubles de CD147 (comprenant son domaine extracellulaire fusionné à la portion Fc des lg) ou d'anticorps dirigés contre le domaine Ig proximal de CD147 bloquent de façon sélective l'adhérence du méningocoque aux cellules endothéliales humaines isolées à partir de capillaires cérébraux (hCMEC/D3) ou de la moelle osseuse (HBMEC). Ceci était observé en conditions statiques comme 


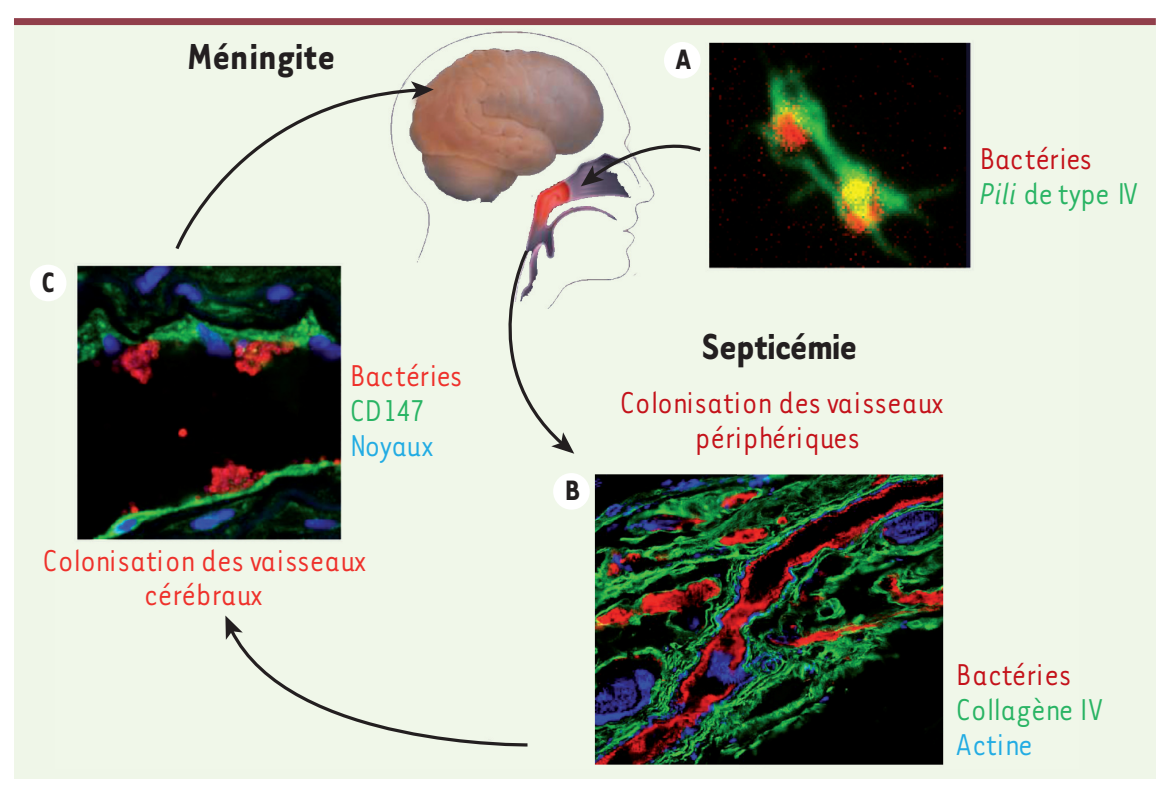

Figure 1. Processus infectieux de N. meningitidis. Le méningocoque est présent de manière asymptomatique au niveau rhinopharyngé chez un grand nombre de sujets sains. Lorsqu'elle atteint la circulation sanguine, cette bactérie se multiplie rapidement, pouvant conduire à des chocs septiques graves. De plus, ces bactéries adhèrent aux cellules endothéliales qui tapissent les vaisseaux par l'intermédiaire de leurs pili de type IV (A). Au niveau de la peau et des muqueuses, l'infection des vaisseaux par le méningocoque crée des lésions hémorragiques (dites purpuriques), dues à des saignements dans les tissus, qui peuvent rapidement évoluer vers une forme grave et souvent mortelle de la maladie (le purpura fulminans) (B). Au niveau du cer-

veau, l'adhérence des méningocoques aux vaisseaux leur permet de franchir la barrière hémato-encéphalique et d'entraîner une méningite lorsqu'ils envahissent les méninges (C). A. Analyse en immunofluorescence des pili de type IV de N. meningitidis. Les corps bactériens (en rouge) portent de nombreux pili de type IV (en vert), longs appendices filamenteux agrégés en faisceaux, qui permettent l'interaction du méningocoque avec les cellules endothéliales humaines (๔ Nawal Maïssa, Institut Cochin). B. Analyse en immunofluorescence de vaisseaux humains, contenus au sein d'une peau humaine greffée sur une souris SCID, infectés par N. meningitidis. Les bactéries (en rouge) ont colonisé les vaisseaux humains délimités par la membrane basale (collagène IV, en vert) et le cytosquelette d'actine (en bleu) (@ Nature Medicine [8]). C. Analyse en immunofluorescence d'une coupe de cerveau humain infectée par $N$. meningitidis. Les bactéries (en rouge) ont colonisé les cellules endothéliales cérébrales qui expriment CD147 (en vert) (en bleu, marquage des noyaux cellulaires) (๔ Nature Medicine [8]).

en conditions dynamiques d'infection en flux laminaire, qui reproduisent les conditions de flux sanguin observées chez l'homme. Enfin, des souches de méningocoque appartenant à des sérogroupes distincts exprimaient ce même phénotype d'adhérence dépendante de CD147, suggérant que le rôle de CD147 dans l'adhérence du méningocoque aux cellules endothéliales était conservé pour les souches pathogènes de méningocoque génétiquement différentes.

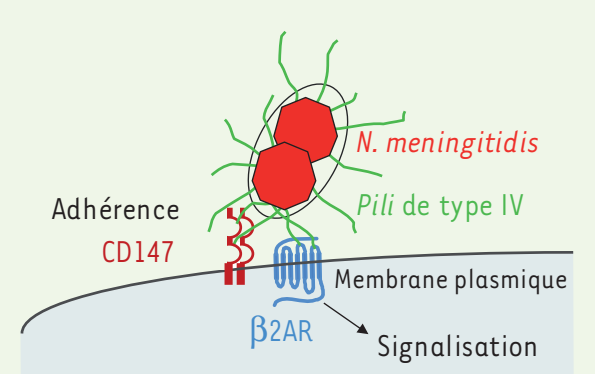

Cellule endothéliale
Les constituants Pilદ et PilV des pili de type IV permettent une interaction directe du méningocoque avec CD147 Les pili de type IV étant indispensables à l'adhérence du méningocoque aux cellules endothéliales par l'intermédiaire de CD147, nous avons recherché s'il existait une interaction directe des pilines composant les pili de type IV avec CDI47. Cette analyse a reposé sur la production des différentes pilines sous forme de protéines recombinantes fusionnées avec la protéine MBP (maltose binding protein). Des expériences d'adhérence du méningocoque aux cellules endothéliales ont montré que seules les pilines «majeure» Pilk et « mineure » PilV étaient capables d'inhiber l'adhérence bactérienne de façon compétitive. Une interaction directe et spécifique des protéines Pilદ et PilV avec la forme soluble de CD147 a été mise en évidence par des expériences de cosédimentation, de transfert d'énergie (Alpha-screen) et de mesures à l'aide d'un microscope à force atomique, indiquant que les forces d'interaction entre les pilines PilE, ou PilV, et CD147 sont d'environ la moitié de celle qui est observée entre CD147 et son ligand naturel, la cyclophiline. Enfin, une analyse en résonance plasmonique de surface a permis de montrer que la faible affinité de chaque molécule de piline pour CD147 était compensée par une forte avidité, due à l'assemblage d'un grand nombre de molécules de piline au sein des fibres de pili de type IV. 
Les ligands bactériens de CD147, PilE et PilV, sont indispensables à la colonisation in vivo des vaisseaux humains par le méningocoque Les infections invasives à méningocoque s'accompagnent fréquemment de localisations cutanées, et la survenue d'un purpura constitue un facteur péjoratif d'évolution de l'infection. Le rôle des ligands de CD147, Pilદ et PilV, dans l'adhérence du méningocoque aux vaisseaux cutanés a pu être exploré grâce à un modèle d'infection récemment développé, constitué de greffons de peau humaine implantés chez des souris SCID (severe combined immunodeficient). Dans ce modèle, les vaisseaux du greffon humain s'anastomosent au réseau sanguin de la souris, permettant l'irrigation des vaisseaux humains par la circulation sanguine de la souris hôte $[10,11]$. Du fait de la stricte spécificité du méningocoque pour l'homme, l'injection par voie parentérale de méningocoques chez la souris conduit à la colonisation sélective des vaisseaux humains du greffon, et à l'apparition consécutive de lésions cutanées similaires à celles qui sont observées chez l'homme (Figure IB). Cette colonisation est spécifiquement due à Pilદ et PilV puisqu'elle n'est pas observée dans les greffons des souris infectées par des bactéries mutantes n'exprimant pas ces pilines.

L'interaction entre les ligands bactériens Pilદ, PilV et CD147 est essentielle

à la colonisation des vaisseaux

cérébraux et des méninges

La méningite est l'une des formes cliniques les plus fréquentes et les plus graves de l'infection invasive à méningocoque. En l'absence de modèles animaux reproduisant la méningite humaine, nous avons utilisé un modèle d'infection in situ sur des coupes de cerveaux humains obtenus chez des sujets décédés d'une cause non infectieuse. Ce modèle a montré que, dans le cerveau, le méningocoque adhérait sélectivement, au niveau des capillaires cérébraux et des méninges, aux seuls types cellulaires exprimant CD147: les cellules endothéliales et les cellules leptoméningées (Figure 1C). Conformément aux résultats obtenus in vitro, seuls les méningocoques exprimant Pilk et PilV étaient capables d'adhérer aux tissus humains. Ce modèle a donc permis de confirmer le rôle du récepteur cellulaire CD147 et des pilines Pilદ et PilV dans l'adhérence du méningocoque au niveau des capillaires cérébraux.

Ce travail a permis d'établir les événements moléculaires précoces par lesquels le méningocoque cible les celIules endothéliales. Cette découverte ouvre des perspectives thérapeutiques majeures pour le traitement et la prévention des dysfonctionnements vasculaires provoqués par ces infections invasives. Notamment, un vaccin prophylactique qui ciblerait l'interaction pili/CD147 serait, en complément des antibiotiques, une thérapie adjuvante attrayante pour limiter les destructions vasculaires liées à l'infection. $\diamond$

CD147 is an essential receptor

for vascular colonization by Neisseria meningitidis

\section{LIENS D'INTÉRÊT}

Les auteurs déclarent n'avoir aucun lien d'intérêt concernant les données publiées dans cet article.

\section{RÉFÉRENCES}

1. Stephens DS, Greenwood B, Brandtzaeg P. Epidemic meningitis, meningococcaemia, and Neisseria meningitidis. Lancet $2007 ; 369$ : 2196-210.

2. Brandtzaeg $P$, van Deuren M. Classification and pathogenesis of meningococcal infections. Methods Mol Biol $2012 ; 799$ : 21-35.

3. Lemichez $\varepsilon$, Lecuit M, Nassif X, Bourdoulous $S$. Breaking the wall: targeting of the endothelium by pathogenic bacteria. Nat Rev Microbiol 2010 ; 8 : 93-104.

4. Melican K, Dumenil G. Vascular colonization by Neisseria meningitidis. Curr Opin Microbiol 2012 ; 15 50-6.

5. Craig L, Li J. Type IV pili: paradoxes in form and function. Curr Opin Struct Biol 2008 ; 18 : 267-77.

6. Coureuil M, Lécuyer $H$, Scott MG, et al. Meningococcus hijacks a beta2-adrenoreceptor/beta-arrestin pathway to cross brain microvasculature endothelium. Cell $2010 ; 143$ : 1149-60.

7. Coureuil M, Marullo S. Récepteur $\beta_{2}$-adrénergique et $\beta$-arrestines : les instruments du méningocoque pour le « casse » des méninges. Med Sci (Paris) $2011 ; 27$ : 365-7.

8. Bernard SC, Simpson N, Join-Lambert 0, et al. Pathogenic Neisseria meningitidis utilizes CD147 for vascular colonization. Nat Med $2014 ; 20$ : 725-31.

9. Iacono KT, Brown AL, Greene MI, Saouaf SJ. CD147 immunoglobulin superfamily receptor function and role in pathology. Exp Mol Pathol 2007 ; 83 : 283-95.

10. Join-Lambert 0 , Lecuyer $H$, Miller F, et al. Meningococcal interaction to microvasculature triggers the tissular lesions of purpura fulminans. J Infect Dis $2013 ; 208: 1590-7$.

11. Melican K, Michea Veloso P, Martin T, et al. Adhesion of Neisseria meningitidis to dermal vessels leads to local vascular damage and purpura in a humanized mouse model. PLoS Pathog 2013 ; 9 : e1003139.

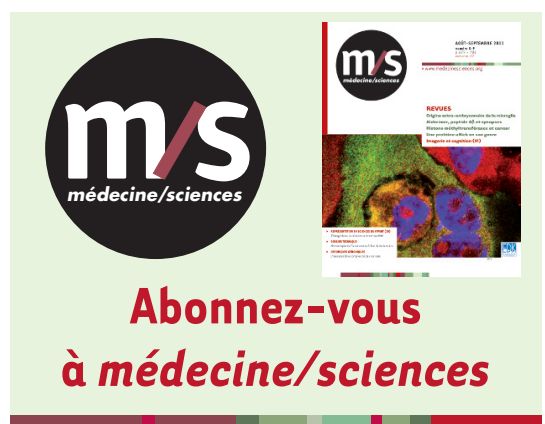

Bulletin d'abonnement page 854 dans ce numéro de $\mathrm{m} / \mathrm{s}$

\section{LA FONDATION PREMUP : UN OPÉRATEUR DE TERRAIN EN PÉRINATALITÉ RECONNU POUR SON EXCELLENCE ET SON INTERDISCIPLINARITÉ}

La Fondation de coopération scientifique PremUp, unique en Europe, intervient sur la prévention FONDATION DE COOPÉRATION SCIENTIFIQUE du handicap à la naissance, par la protection de la santé de la femme enceinte et du nouveau-né.
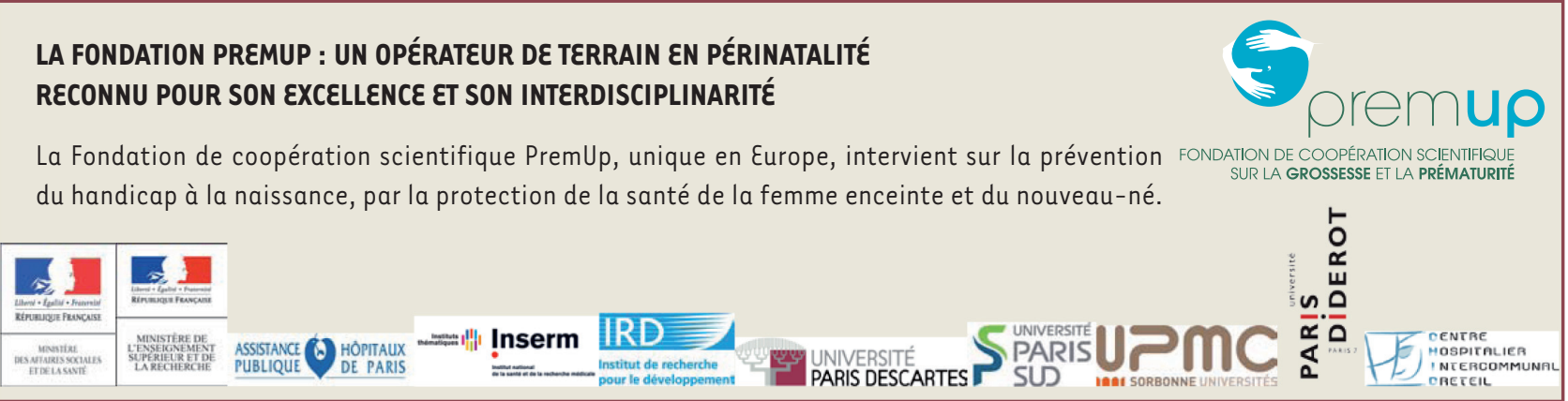